Documento No. 30

La Estabilidad de la Demanda de Dinero en México

por

Guillermo Ortiz

Noviembre, 1980

Las ideas contenidas en el presente ensayo son responsabilidad exclusiva del autor y no reflejan la posición del Banco de México. 


\title{
LA ESTABILIDAD DE LA DEMANDA DE DINERO EN MÉXICO
}

\author{
por \\ Guillermo Ortiz*
}

Introducción.

La existencia de relaciones agregadas estables es, desde luego, una condición necesaria para la construcción de modelos macroeconómicos útiles para la evaluación de políticas económicas alternativas. Se dice que una función es estable si se puede establecer una relación causal entre una variable dependiente y un número reducido de variables predeterminadas o exógenas que sea consistente a través del tiempo.

Existen varias maneras de realizar pruebas econométricas sobre la estabilidad temporal de una relación. Una de las más conocidas consiste en efectuar análisis de covarianza de los residuos correspondientes a dos períodos de tiempo distintos, con el objeto de verificar si los coeficientes estimados en ambos períodos pertenecen a la misma población. Una limitante de este tipo de prueba es que los períodos deben escogerse a priori, en base a cierto conocimiento previo de la información ${ }^{1}$.

Otro tipo de pruebas de estabilidad tiene que ver con la capacidad del modelo utilizado para realizar pronósticos aceptables dentro del período muestral, medidos estos por la magnitud de la raíz del error medio cuadrado de la simulación. Si la ecuación utilizada contiene una variable dependiente rezagada -como en la mayoría de las estimaciones que se han realizado sobre la demanda de dinero en el corto plazo- los pronósticos obtenidos pueden ser de carácter estático o dinámico.

Una tercera prueba de estabilidad, que ha sido utilizada en fechas recientes en estimaciones sobre demanda de dinero, se basa también en la magnitud de los errores de

\footnotetext{
* Agradezco la valiosa ayuda de Víctor J. Pérez.

${ }^{1}$ Este método, conocido como la prueba de Chow, está ampliamente explicado en Johnston (1972).
} 
predicción. Esta prueba, que se debe a Brown, Durbin y Evans (1975), tiene la ventaja que puede aplicarse a casi todo el período muestral, por lo que se pueden detectar inestabilidades sin necesidad de realizar una división previa y arbitraria de dicho período.

En el caso de la demanda de dinero, prácticamente todos los estudios empíricos realizados para México concluyen que la demanda de dinero es efectivamente una función estable del ingreso y de la tasa de inflación, variable esta última que representa el costo de oportunidad de mantener saldos monetarios. Siendo que los períodos que cubren estos estudios son muy diversos, parecería que existe una amplia evidencia sobre la estabilidad de dicha relación. Sin embargo, la mayor parte de los autores no realizan ninguna prueba formal de estabilidad, y sus conslusiones se basan únicamente en la obtención de coeficientes estadísticamente significativos que muestran el signo esperado.

En este trabajo se explora más a fondo el comportamiento de la demanda por dinero a nivel trimestral de 1960 a 1979 en México, y se efectúan distintas pruebas de estabilidad. 


\section{Simulaciones Estáticas y Dinámicas.}

En un trabajo reciente, donde se ensayaron diversas formulaciones empíricas de la demanda por dinero, se encontró que la ecuación

$$
\begin{aligned}
\ln \left(\frac{M_{1}}{P}\right) & =\alpha_{0}+\alpha_{1} \ln Y_{t}^{c}+\alpha_{2} \operatorname{lnRL_{t}+\alpha _{3}\operatorname {ln}\pi } \pi_{.2 t} \\
& +\alpha_{4} \ln \mathrm{dtc}+\alpha_{5} \ln \frac{M_{1}}{P}{ }_{t-1}+\mathrm{d}_{1}+\mathrm{d}_{2}+\mathrm{d}_{3}+\epsilon_{t}
\end{aligned}
$$

donde

$$
\begin{aligned}
& \left(M_{1} / P\right)=\text { billetes y monedas; } \\
& \mathrm{Y}_{t}^{c} \quad=\text { ingreso corriente (real); } \\
& \mathrm{RL}_{t}=\text { tasa de rendimiento promedio sobre pasivos líquidos; } \\
& \pi_{.2 t}=\text { tasa de inflación medida por el índice de precios al consumidor } \\
& {\left[\pi=.2\left(\mathrm{P}_{t} / \mathrm{P}_{t-1}\right)+.8\left(\mathrm{P}_{t-1} / \mathrm{P}_{t-2}\right)\right]} \\
& \text { dtc = diferencia entre el tipo de cambio real y el observado; } \\
& \epsilon_{t} \quad=\text { error aleatorio; }
\end{aligned}
$$$$
\mathrm{d}_{1}, \mathrm{~d}_{2}, \mathrm{~d}_{3}=\text { variables "dummy" para corregir por variaciones estacionarias. }
$$

resultó la mejor especificación funcional desde el punto de vista econométrico ${ }^{1 /}$. En el cuadro 1 se resumen las estimaciones trimestrales efectuadas con dicha ecuación.

1/ G. Ortiz (1980). 


\section{Cuadro 1}

Estimaciones de la Ecuación (1)

Variable Dependiente $\mathrm{M}_{1} / \mathrm{P}$

\begin{tabular}{|c|c|c|c|c|c|c|c|c|c|}
\hline PERIODO & $\mathrm{Y}_{t}^{c}$ & $\mathrm{RL}_{t}$ & $\pi_{.2 t}$ & $\mathrm{dtc}$ & $\left(\mathrm{M}_{1} / \mathrm{P}\right)_{t-1}$ & $\mathrm{R}^{2}$ & Durbin-h & Rho & $\begin{array}{l}\text { Error } \\
\text { Estandad }\end{array}$ \\
\hline $1960-1972$ & $\begin{array}{c}.277^{*} \\
(2.65)\end{array}$ & $\begin{array}{l}-.227^{*} \\
(-2.39)\end{array}$ & $\begin{array}{l}-.0017 \\
(-.624)\end{array}$ & $\begin{array}{l}.0023 * \\
(2.38)\end{array}$ & $\begin{array}{l}.758^{*} \\
(6.85)\end{array}$ & .993 & 1.45 & -.348 & .021 \\
\hline $\begin{array}{c}\text { 1960-1976 } \\
\text { III }\end{array}$ & $\begin{array}{r}.077 \\
(1.12)\end{array}$ & $\begin{array}{l}-.104 * \\
(-2.25)\end{array}$ & $\begin{array}{c}-.0012^{*} \\
(-2.31)\end{array}$ & $\begin{array}{r}-.0012 \\
(-.76)\end{array}$ & $\begin{array}{l}.954^{*} \\
(13.9)\end{array}$ & .995 & 1.13 & -.447 & .023 \\
\hline $1960-1979$ & $\begin{array}{c}.105^{*} \\
(1.87) \\
\end{array}$ & $\begin{array}{l}-.085^{*} \\
(-2.38) \\
\end{array}$ & $\begin{array}{r}-.0019 * \\
(-5.96) \\
\end{array}$ & $\begin{array}{r}-.00011^{*} \\
(-8.58) \\
\end{array}$ & $\begin{array}{c}.935^{*} \\
(16.48) \\
\end{array}$ & .995 & 1.20 & -.513 & .025 \\
\hline
\end{tabular}

* Significativas al 5\%. Valores t entre paréntesis. Las variables dummies resultaron todas altamente significativas.

Fuente: G. Ortiz (1980).

Como puede observarse, todos los coeficientes muestran el signo esperado y son estadísticamente significativos a un nivel de 5\%, a excepción del coeficiente correspondiente al ingreso que se deteriora considerablemente cuando se pasa del período estable (1960-1972) al período inflacionario pre-devaluación (1960-1976 III). Se aprecia también en el cuadro 1 que el coeficiente de la tasa de interés disminuye de manera considerable conforme se pasa de un período al siguiente, mientras que se fortalece el correspondiente a las expectativas inflacionarias. Sin embargo, el orden de magnitud de los coeficientes de esta última variable es sustancialmente menor a los correspondientes a la tasa de interés. De esto puede concluirse, en contraste con resultados empíricos obtenidos en trabajos previos sobre México, que cambios en la inflación esperada influyen de manera menos significativa en la demanda de dinero que modificaciones en la tasa de interés pasiva. Este resultado parece ser bastante robusto ya que se obtuvo después de ensayar con diferentes procedimientos para calcular la inflación esperada, incluyendo uno de expectativas racionales ${ }^{1}$.

\footnotetext{
${ }^{1 /}$ Véase el trabajo mencionado anteriormente.
} 
Por otra parte, la velocidad de ajuste también se reduce considerablemente en el período inflacionario. Mientras que durante el lapso 1960-1972 aproximadamente el 66 por ciento del ajuste a los saldos reales deseados se realizaba en un año, en el período 1960-1979 se llevara a cabo sólo el 24 por ciento de dicho ajuste. Esta reducción en la velocidad de ajuste compensó parcialmente el deterioro de los coeficientes del ingreso y de la tasa de interés, de manera que las elasticidades de largo plazo experimentaron una menor variación que las de corto plazo como se aprecia a continuación.

\section{CUADRO 2 \\ ELASTICIDADES DE LARGO PLAZO}

\begin{tabular}{lccc}
\hline PERIODO & $\mathrm{Y}_{t}^{c}$ & $\mathrm{RL}_{t}$ & $\pi_{.2 t}$ \\
\hline $1960-1972$ & 1.14 & .93 & $.007^{*}$ \\
$1960-1976 \mathrm{III}$ & $1.67^{*}$ & 2.26 & .026 \\
$1960-1979$ & 1.41 & 1.30 & .029 \\
\hline
\end{tabular}

*No significativo (al 5\%).

Debido a la reducción de la velocidad de ajuste, las elasticidades de la demanda de dinero con respecto al ingreso y a la tasa de interés aumentan en vez de disminuir. Esta reducción puede obedecer al hecho que la mayor incertidumbre asociada con un ritmo mas alto de crecimiento de los precios provoca una reacción de ajuste más lenta de parte del público. A su vez, como se explica en el trabajo mencionado anteriormente, el aumento de elasticidad ingreso puede deberse también a que el efecto de la mayor incertidumbre causada por la inflación (que tiende a aumentar la demanda de dinero por transacciones) es mayor que el efecto del alza en el costo de oportunidad de mantener dinero (que, por el contrario, tiende a reducir la demanda).

Los fuertes cambios observados en el tamaño de los coeficientes cuando se pasa de un período de estimación al siguiente, plantean dudas sobre la estabilidad temporal de la demanda de dinero. A continuación se reproducen los resultados de la prueba de Chow que se aplicó a la ecuación 1 . 


\section{CUADRO 3}

Estadístico-F para $\mathrm{H}_{o}$ : Los Coeficientes son

Iguales en dos períodos muestrales

\begin{tabular}{llcc}
\hline PERIODOS MUESTRALES & \multicolumn{2}{l}{ Valores Críticos de F } & F-Calculada \\
& $1 \%$ & $5 \%$ & \\
\hline $\begin{array}{l}1960-1972 / \\
1960-1976 \text { III }\end{array}$ & 3.13 & 2.20 & 1.543 \\
F $(16,43)$ & & & \\
$1960-1972 /$ & & & \\
$1960-1979$ & 2.30 & 1.81 & 2.018 \\
F $(28,46)$ & & & \\
$1960-1976$ III/ & & & 2.235 \\
$1960-1979$ & 3.34 & 2.32 & \\
F $(13,55)$ & & & \\
\hline
\end{tabular}

Fuente: Cuadro 10 de G. Ortiz (1980).

Pese a la variación experimentada por los coeficientes, la hipótesis de que estos coeficientes pertenecen a la misma población de puede ser rechazada al nivel de confianza del uno por ciento en ninguno de los tres casos. Al nivel de 5 por ciento, sólo al comparar los períodos 1960-1972/1960-1979 se detecta la presencia de un cambio estructural, ya que el valor de la F calculada excede al valor crítico correspondiente. Por lo tanto, atendiendo a este criterio de estabilidad, la evidencia no es conclusiva, aunque sí sorprende el hecho que no se haya detectado una inestabilidad más marcada.

Con el objeto de explorar más a fondo el problema de estabilidad de la demanda de dinero, y de evaluar la posibilidad de realizar buenos pronósticos fuera del período muestral, se realizaron simulaciones estáticas y dinámicas. En primer término, la ecuación (1) se estimó nuevamente para el período 1960-1972, habiéndose obtenido el siguiente resultado ${ }^{1 /}$ :

\footnotetext{
${ }^{1 /}$ Los coeficientes de $(1$ ') difieren ligeramente de los presentados en el cuadro 1 debido a que se realizaron algunos ajustes menores a la base de datos.
} 


$$
\begin{aligned}
& \ln \left(\frac{M_{1}}{P}\right)=-.195+.248 \ln \mathrm{Y}_{t}^{c}-.233 \ln \mathrm{RL}_{t} \\
& (-1.81) \quad(2.35) \quad(-2.46) \\
& -.007 \ln \pi_{.2}+.019 \mathrm{dtc}--.188 \mathrm{~d}_{1} \\
& \begin{array}{lll}
(-.725) & (1.91) \quad(-15.87)
\end{array} \\
& \begin{array}{llll}
-.147 d_{2} & -.131 d_{3} & +.789
\end{array} \\
& (-17.38) \quad(7.10)
\end{aligned}
$$

$\mathrm{R}^{2}=.993 \quad$ Durbin- $\mathrm{h}=1.23 \quad \mathrm{Rho}=-.349 \quad$ Error Estandard $=.022$

El signo del coeficiente de la variable dtc (diferencial entre el tipo de cambio real y el observado), que se utiliza como una proxy para medir expectativas de devaluación, es contrario al esperado en este período.

Sin embargo, como puede observarse en el cuadro 1, dicho coeficiente se torna negativo cuando se pasa a los períodos inflacionarios en los que, lógicamente, se agudiza la incertidumbre sobre el valor futuro del tipo de cambio. Por esta razón, se decidió incluir dicha variable en la regresión. En la gráfica 1 se ilustra el comportamiento histórico de la serie de medio circulante así como el calculado mediante la ecuación (1'). Como puede observarse en esta gráfica, la serie presenta una fuerte estacionalidad, por lo que fue necesario utilizar tres variables "dummy" a fin de captar la reducción en el nivel de los saldos de los tres primeros trimestres del año con respecto al mes de diciembre anterior.

En el cuadro 4 se presentan los resultados de simular estáticamente la ecuación (1') de 1973 a 1979.

Como puede apreciarse, los resultados de las simulaciones son bastantes satisfactorias. El error de simulación como porcentaje de la variable dependiente es muy reducido y sólo en un trimestre (1976-I) dicho error excede del uno por ciento de la variable dependiente; el promedio de los errores como proporción de la variable dependiente es de sólo .46 por ciento. Para comparar estos resultados, conviene revisar los obtenidos en un trabajo reciente por Hein (1980), quien estimó una ecuación de demanda de dinero para 
Estados Unidos (utilizando una especificación ensayada previamente por Goldfeld (1973) y otros autores) con datos de 1960-IV a 1974-II, realizando posteriormente simulaciones estáticas y dinámicas para el período 1974-III a 1979-IV. La simulación estática arrojó un error promedio de 5.85 por ciento de la variable dependiente, cifra bastante mayor a la obtenida con datos mexicanos. La gráfica 2 ilustra el comportamiento de esta simulación.

Gráfica 1

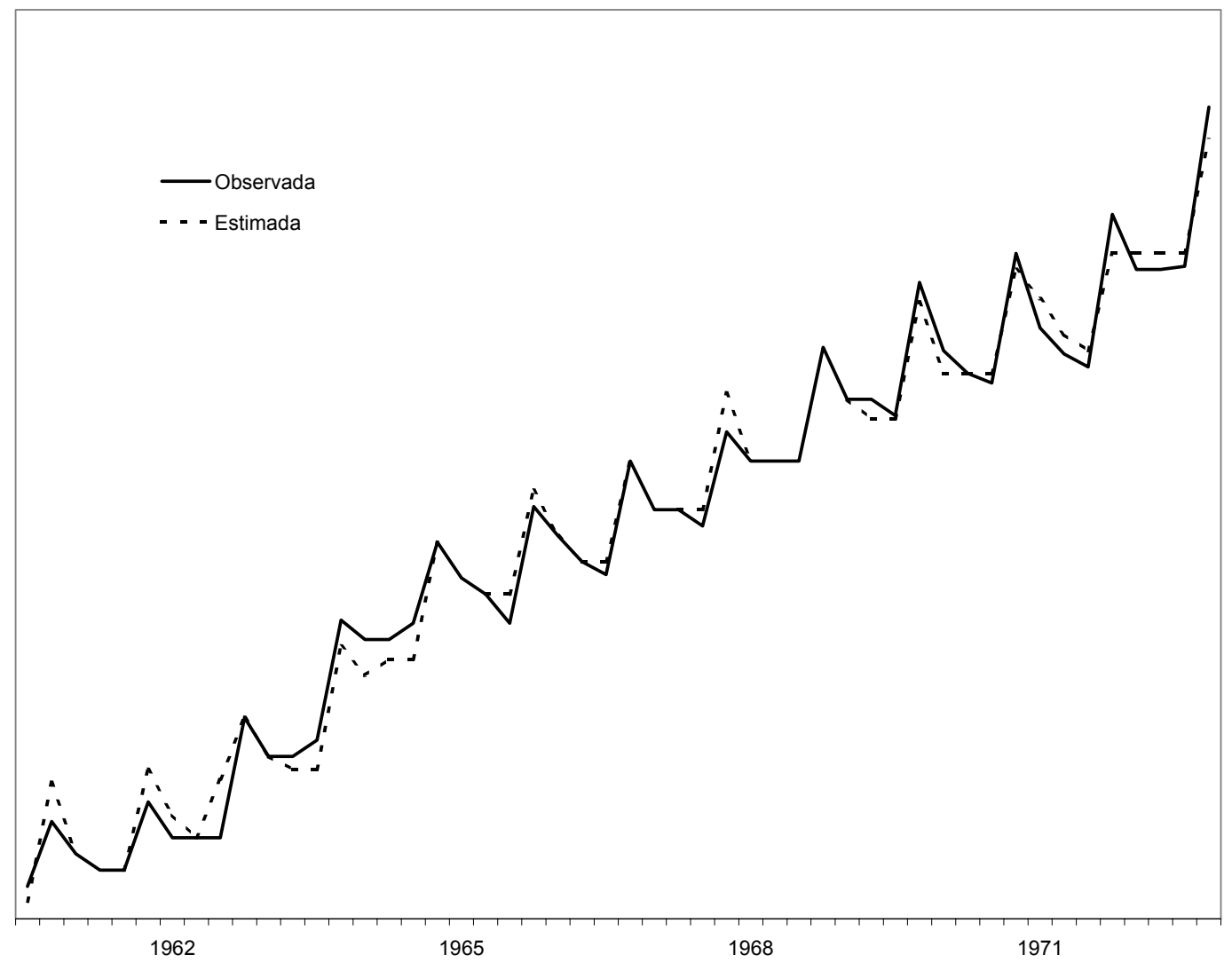




\section{CUADRO 4}

SIMULACIONES ESTATICAS DE LA DEMANDA DE DINERO

(1973-I - 1979-IV)

\begin{tabular}{|c|c|c|c|c|c|}
\hline PERIODO & $\begin{array}{l}\ln \left(\mathrm{M}_{1} / \mathrm{P}\right) \\
\text { observada }\end{array}$ & $\begin{array}{l}\ln \left(\mathrm{M}_{1} / \mathrm{P}\right) \\
\text { simulada }\end{array}$ & $\begin{array}{c}\text { Error } \\
\text { de } \\
\text { Predicción } \\
\text { Estática }\end{array}$ & $\begin{array}{c}\text { Error } \\
\text { como } \\
\text { Porcentaje } \\
\text { de } \\
\text { Variable } \\
\text { Dependiente }\end{array}$ & $\begin{array}{l}\text { Error de } \\
\text { Proyección } \\
\text { de balances } \\
\text { reales en } \\
\text { miles de } \\
\text { millones } \\
\end{array}$ \\
\hline $\mathrm{I} / 1973$ & 10.5746 & 10.5850 & -.01035 & -.09 & -.409 \\
\hline II/1973 & 10.5812 & 10.5643 & .01694 & .16 & .660 \\
\hline III/1973 & 10.5693 & 10.5595 & .00984 & .08 & .379 \\
\hline IV/1973 & 10.7286 & 10.6985 & .03009 & .27 & 1.353 \\
\hline I/1974 & 10.6015 & 10.6614 & -.05985 & -.55 & -2.481 \\
\hline II/1974 & 10.6034 & 10.5948 & .08615 & .81 & .344 \\
\hline III/1974 & 10.5672 & 10.6123 & -.04517 & -.42 & -1.791 \\
\hline IV/1974 & 10.7126 & 10.7242 & -.01156 & -.10 & -.524 \\
\hline $\mathrm{I} / 1975$ & 10.6317 & 10.6566 & -.02492 & -.22 & -1.044 \\
\hline II/1975 & 10.6185 & 10.6702 & -.05171 & -.48 & -2.169 \\
\hline III/1975 & 10.5666 & 10.6660 & -.09991 & -.94 & -4.056 \\
\hline IV/1975 & 10.7517 & 10.7580 & -.06279 & -.57 & -.295 \\
\hline $\mathrm{I} / 1976$ & 10.6413 & 10.7807 & -.13942 & -1.31 & -6.256 \\
\hline II/1976 & 10.6566 & 10.7228 & -.06618 & -.62 & -2.906 \\
\hline III/1976 & 10.6776 & 10.6519 & .02577 & .24 & 1.100 \\
\hline IV/1976 & 10.8296 & 10.7939 & .03072 & .28 & 1.770 \\
\hline $\mathrm{I} / 1977$ & 10.6426 & 10.7403 & -.09773 & -.91 & -4.298 \\
\hline II/1977 & 10.5930 & 10.6436 & -.05052 & -.47 & -2.068 \\
\hline III/1977 & 10.5526 & 10.6322 & -.07962 & -.75 & -3.171 \\
\hline IV/1977 & 10.7825 & 10.7450 & .03756 & .34 & 1.772 \\
\hline $\mathrm{I} / 1978$ & 10.6923 & 10.7658 & -.07354 & -.68 & -3.357 \\
\hline II/1978 & 10.7125 & 10.7573 & -.04484 & -.41 & -2.057 \\
\hline III/1978 & 10.6936 & 10.7877 & -.09406 & -.87 & -4.348 \\
\hline IV/1978 & 10.9046 & 10.8941 & .01054 & .09 & .568 \\
\hline I/1979 & 10.8338 & 10.8888 & -.05492 & -.50 & -2.866 \\
\hline II/1979 & 10.8495 & 10.8634 & -.01393 & -.12 & .721 \\
\hline III/1979 & 10.8210 & 10.8633 & -.04230 & -.39 & -2.163 \\
\hline IV/1979 & 11.0100 & 10.9314 & .07860 & .72 & 4.571 \\
\hline
\end{tabular}

Estadísticas de la Simulación Estática:

Error medio al cuadrado (RMSE) $\quad .0578$

Error medio absoluto (MAE) $\quad .04708$

Coeficiente de Desigualdad de Theil $\quad .002703$ 
Fracción del error debido a
i) sesgo
ii) variación
iii) co-variación

Simulaciones Estáticas de la Demanda de Dinero $\left(\mathrm{M}_{1}\right)$

(1973-I/1979-IV)

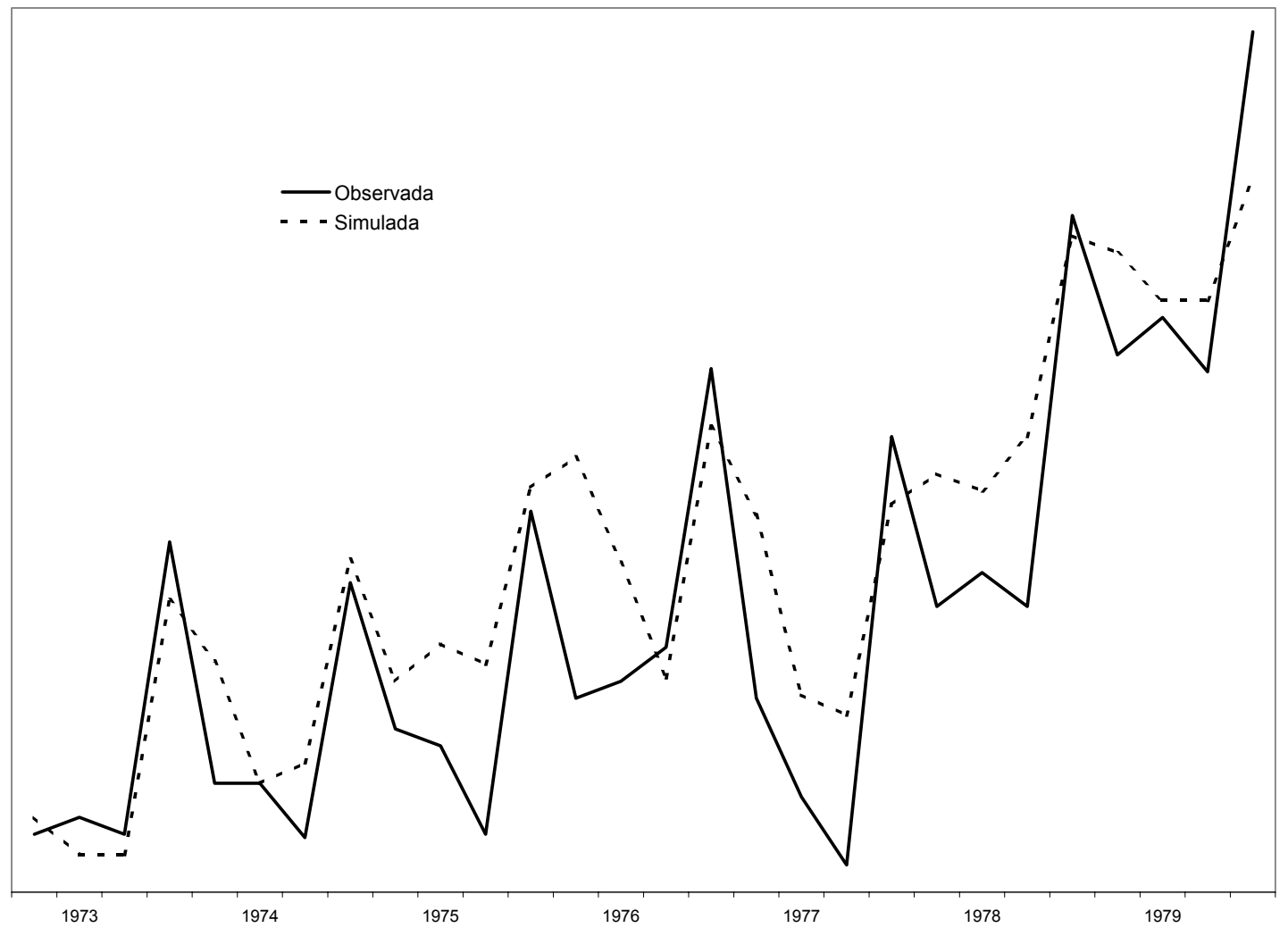

Otro aspecto que debe resaltarse del ejercicio estático es que no se observa una tendencia de la simulación a subestimar o sobrestimar de manera consistente la variable dependiente. De hecho, el 37.5 por ciento de los valores simulados está por debajo del valor real correspondiente, mientras que el porcentaje restante se encuentra por encima de éste ${ }^{1 /}$. De la gráfica 2 es también aparente que la ecuación utilizada sigue con bastante precisión los cambios marcados por el comportamiento de $\mathrm{M}_{1}$; en particular, la simulación rastrea

\footnotetext{
1/ En contraste, las simulaciones realizadas por Hein (1980) muestran una tendencia a sobrestimar de manera consistente la demanda de dinero. Este comportamiento de las simulaciones, que ya había sido detectado por Goldfeld (1976), ha suscitado una extensa controversia en la literatura especializada ya que varios autores sostienen que la demanda de dinero sufrió un desplazamiento en 1974 (debido a la introducción de diversos instrumentos monetarios y a innovaciones tecnológicas). Véase por ejemplo los artículos de García y Pak (1979) y Porter, R., Simpson, T. Y Mauskopf, E. (1979).
} 
aceptablemente el brusco aumento de los saldos reales en diciembre de 1976 causado por la devaluación, y también sigue de cerca la rápida caída experimentada en los primeros trimestres de 1977 (que obedeció seguramente a la dolarización y a los rápidos aumentos del nivel de precios).

El cuadro 5 muestra los resultados de la simulación dinámica; al igual que para el ejercicio anterior, se estimó la ecuación (1) de 1960 a 1972, y se proyectó de 1973-I a 1979-IV. En el primer período de la simulación, se utilizó la variable dependiente rezagada real, por lo que coinciden los valores de las proyecciones estáticas y dinámicas en el primer trimestre de 1973. En las estimaciones subsecuentes, el valor de la variable dependiente rezagada utilizada en el período t es la generada por la misma proyección en el período $\mathrm{t}-1$, de manera que cabe esperar un menor grado de precisión de los resultados obtenidos en este tipo de ejercicio. Es claro que al utilizar variables rezagadas generadas por los mismos pronósticos de la simulación mo existe un mecanismo de autocorrección, por lo que la trayectoria de los errores tenderá a acumularse recursivamente a través del tiempo ${ }^{2}$.

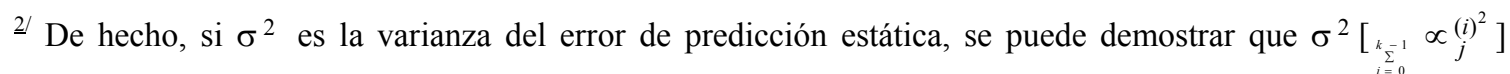
corresponde a la varianza del error de proyección dinámica en el período $\mathrm{T}+\mathrm{k}$, donde $\propto_{j}$, representa el coeficiente de la variable dependiente rezagada. Véase a Hein (1980). 


\section{CUADRO 5}

\section{SIMULACIONES DINAMICAS DE LA DEMANDA DE DINERO $\left(\mathrm{M}_{1}\right)$}

$$
\text { (1973-I - 1979-IV) }
$$

\begin{tabular}{|c|c|c|c|c|c|}
\hline PERIODO & $\begin{array}{l}\ln \left(\mathrm{M}_{1} / \mathrm{P}\right) \\
\text { observada }\end{array}$ & $\begin{array}{l}\ln \left(\mathrm{M}_{1} / \mathrm{P}\right) \\
\text { simulada }\end{array}$ & $\begin{array}{c}\text { Error } \\
\text { de } \\
\text { Predicción } \\
\text { Dinámica }\end{array}$ & $\begin{array}{c}\text { Error } \\
\text { como } \\
\text { Porcentaje } \\
\text { de } \\
\text { Variable } \\
\text { Dependiente }\end{array}$ & $\begin{array}{l}\text { Error de } \\
\text { Proyección } \\
\text { de balances } \\
\text { reales en } \\
\text { miles de } \\
\text { millones } \\
\end{array}$ \\
\hline $\mathrm{I} / 1973$ & 10.5746 & 10.5850 & -.0103 & -.09 & -.409 \\
\hline II/1973 & 10.5812 & 10.5725 & .0087 & .00 & .341 \\
\hline III/1973 & 10.5693 & 10.5461 & .0232 & .21 & .892 \\
\hline IV/1973 & 10.7286 & 10.6907 & .0378 & .35 & 1.697 \\
\hline $\mathrm{I} / 1974$ & 10.6015 & 10.6376 & -.0361 & -.34 & -1.477 \\
\hline II/1974 & 10.6034 & 10.6421 & -.0386 & -.36 & -1.589 \\
\hline III/1974 & 10.5672 & 10.6055 & -.0383 & -.36 & -1.566 \\
\hline IV/1974 & 10.7126 & 10.7598 & -.0472 & -.44 & -2.171 \\
\hline $\mathrm{I} / 1975$ & 10.6317 & 10.6658 & -.0340 & -.31 & -1.437 \\
\hline II/1975 & 10.6185 & 10.6898 & -.0713 & -.67 & -3.021 \\
\hline III/1975 & 10.5666 & 10.7079 & -.1407 & -1.33 & -5.891 \\
\hline IV/1975 & 10.7517 & 10.8368 & -.0851 & -.79 & -4.149 \\
\hline $\mathrm{I} / 1976$ & 10.6413 & 10.7857 & -.1443 & -1.35 & -6.497 \\
\hline II/1976 & 10.6566 & 10.8328 & -.1762 & -1.65 & -8.183 \\
\hline III/1976 & 10.6776 & 10.7041 & -.0264 & -.24 & -1.164 \\
\hline IV/1976 & 10.8246 & 10.7736 & .0510 & .47 & 2.498 \\
\hline $\mathrm{I} / 1977$ & 10.6426 & 10.7161 & -.0734 & -.68 & -3.194 \\
\hline II/1977 & 10.5930 & 10.7207 & -.1276 & -1.20 & -5.428 \\
\hline III/1977 & 10.5526 & 10.6721 & -.1195 & -1.13 & -4.858 \\
\hline $\mathrm{IV} / 1977$ & 10.7825 & 10.8078 & -.0252 & -.23 & -1.234 \\
\hline $\mathrm{I} / 1978$ & 10.6923 & 10.7362 & -.0439 & -.41 & -1.975 \\
\hline II/1978 & 10.7125 & 10.8154 & -.1028 & -.95 & -4.867 \\
\hline III/1978 & 10.6936 & 10.8231 & -.1294 & -1.21 & -6.093 \\
\hline IV/1978 & 10.9046 & 10.9683 & -.0636 & -.58 & -3.634 \\
\hline $\mathrm{I} / 1979$ & 10.8338 & 10.8804 & -.0466 & -.43 & -2.418 \\
\hline II/1979 & 10.8495 & 10.9068 & -.0572 & -.52 & -3.037 \\
\hline III/1979 & 10.8210 & 10.8743 & -.0533 & -.49 & -2.740 \\
\hline IV/1979 & 11.0100 & 10.9622 & .0478 & .43 & 2.822 \\
\hline
\end{tabular}

Estadísticas de la Simulación Dinámica:

$\begin{array}{ll}\text { Error medio al cuadrado (RMSE) } & .0796 \\ \text { Error medio absoluto (MAE) } & .06646 \\ \text { Coeficiente de Desigualdad de Theil } & .003716\end{array}$

Fracción del error debido a 

i) sesgo

Pese a estos problemas, los resultados reportados en el cuadro 5 aparentemente son muy buenos. El error máximo de la proyección para un período apenas llega al 1.65 por ciento de la variable dependiente, y sólo en 6 de los 22 valores simulados dicho error excede al uno por ciento.

El error promedio es de .61 por ciento de los saldos reales; aun que mayor que el obtenido en la proyección estática es, sin embargo, de magnitud bastante pequeña. Esta simulación se ilustra en la gráfica 3 .

Gráfica 3

Simulaciones Dinámicas de la Demanda de Dinero

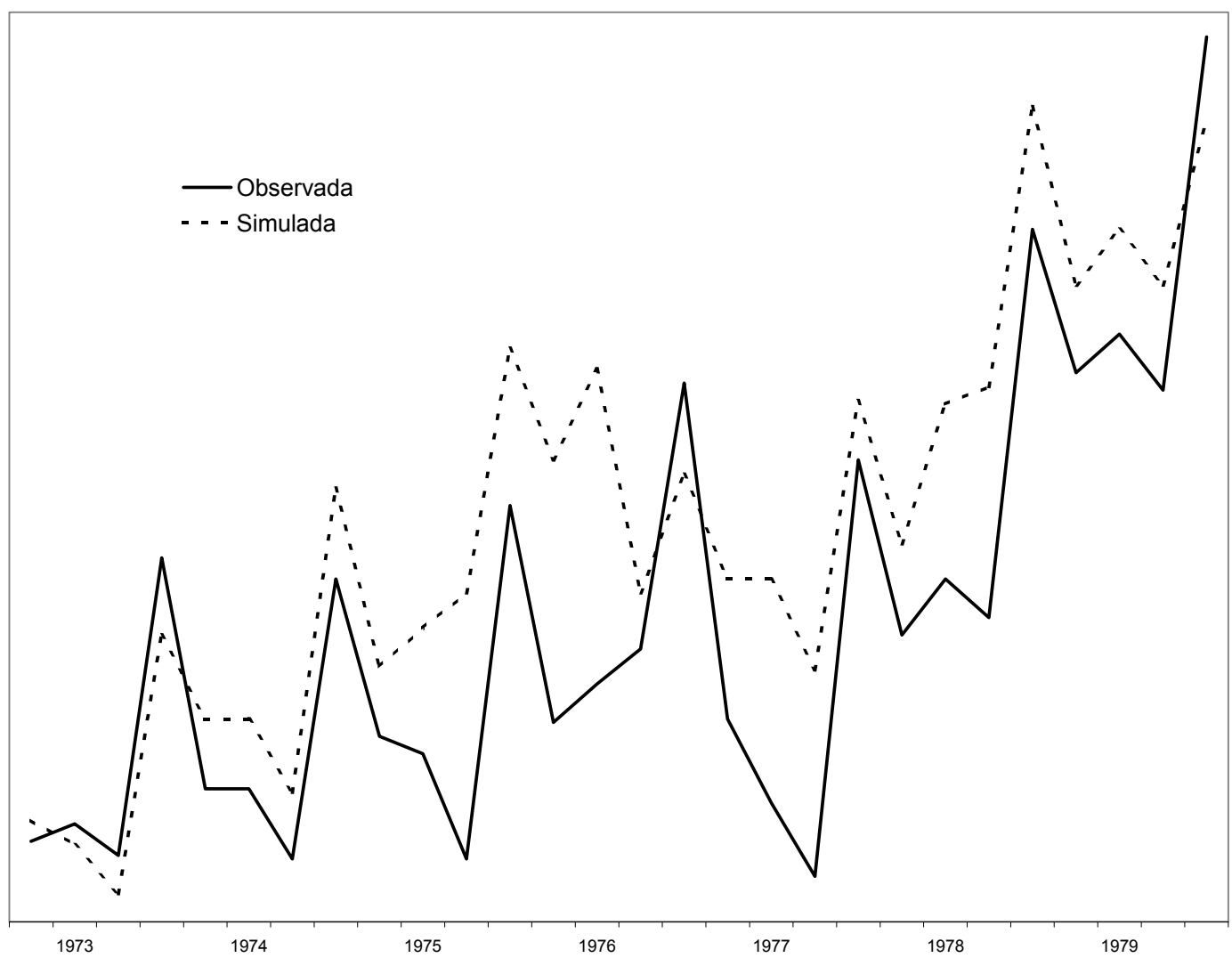


Debe anotarse, asimismo, que a diferencia de la estática, la simulación dinámica de la ecuación (1) tiende a sobrestimar de manera casi consistente la demanda de dinero. En el cuadro 5 puede apreciarse que sólo en 5 de las 28 observaciones el valor simulado está por debajo del observado.

Por otra parte, a fin de tener una base de comparación, conviene nuevamente contrastar estos resultados con los correspondientes a la simulación dinámica realizada por Hein en el trabajo citado anteriormente. El error promedio obtenido por este autor es superior al 11 por ciento de la variable dependiente, y para los cuatro trimestres de 1979, dicho error es mayor al 18 por ciento ${ }^{1}{ }^{\prime}$.

En resumen, los resultados de las simulaciones estática y dinámica sugieren que la especificación de la demanda por dinero formulada en la ecuación (1) es bastante estable en el período considerado.

\footnotetext{
${ }^{1 /}$ La magnitud de este error aparentemente se debe a problemas de especificación de la ecuación a partir de 1974. En períodos muestrales anteriores, las simulaciones dinámicas producen resultados bastante mejores. Por ejemplo, Goldfeld (1973) estimó una ecuación (,uy parecida a la utilizada posteriormente por Hein) para el período 1952-II a 1961_IV e hizo la extrapoli dinámicamente hasta 1972-IV obteniendo raíces de los errores medios cuadrados en la simulación (RMSE) de aproximadamente el doble de tamaño que el error estándar de estimación. El valor del cociente RMSE/error estándar en el trabajo de Hein es superior a 10, mientras que el correspondiente a la simulación dinámica de la ecuación (1) es de 3.7.
} 


\section{Una Prueba Alternativa.}

Un método mas formal para evaluar la constancia de los parámetros de una regresión a través del tiempo, llamado la prueba de Brown, Durbin y Evans (BDE) o de la "suma cumulativa de los residuales recursivos" (CUSUM), ha sido aplicada recientemente en algunos trabajos sobre demanda de dinero ${ }^{1 /}$.

Para aplicar esta prueba, se deben calcular pronósticos un período adelante para $\mathrm{r}=\mathrm{k}+1, \ldots \mathrm{T}$ períodos, utilizando una regresión con $\mathrm{r}-1$ observaciones, en la que $\mathrm{k}$ es el número de regresores (incluyendo la constante y las variables "dummy”) y $\mathrm{T}$ es el tamaño de la muestra. En el caso de la ecuación 1, k es igual a 9, de manera que la primera predicción se basa en una regresión estimada sobre la muestra 1 a 10, y así sucesivamente. Una vez que se han elaborado las predicciones hasta $\mathrm{T}$, se toman los errores cuadrados de cada una de éstas y se calcula el siguiente estadístico:

$$
\mathrm{S}_{r}=\left(\sum_{k+1}^{r} w_{t}^{2}\right) /\left(\sum_{R+1}^{T} w_{t}^{2}\right) \quad \mathrm{r}=\mathrm{k}+1, \ldots \ldots \mathrm{T}
$$

Donde las $\mathrm{w}_{t}^{2}$ representan precisamente los errores cuadrados de predicción un período adelante. El denominador consiste, por lo tanto es la sumatoria del cuadrado de los errores en todo el período muestral, y el estadístico de $\mathrm{S}_{r}$ toma valores entre 0 y 1 .

\footnotetext{
1/ Brown, Durbin y Evans (1975). Véase también los trabajos de Khan (1974), Heller y Khan (1979), Cockerline (1979), Hafer y Hein (1980) y Valdez (1980).
} 
La hipótesis nula de la prueba es que los coeficientes de la regresión son constantes a través del tiempo, de manera que los valores calculados de $\mathrm{S}_{r}$ deben caer alrededor de su valor esperado $\left(\mathrm{E}\left(\mathrm{S}_{r}\right)=(\mathrm{r}-\mathrm{k}) /(\mathrm{T}-\mathrm{k})\right)$. Gráficamente, se pueden establecer límites de confianza paralelos al valor medio, $\mathrm{S}_{r}{ }_{ \pm} \mathrm{C}_{o}+(\mathrm{r}-\mathrm{k}) /(\mathrm{T}-\mathrm{k})$, de tal modo que si la trayectoria muestral de $\mathrm{S}_{r}$ sale de estas bandas de confianza, se rechaza la hipótesis nula de constancia de los coeficientes ${ }^{1}$. La gráfica 4 ilustra esta prueba.

\section{Gráfica 4}

Prueba de Brown, Durbin y Evans

(Ecuación 1; variable dependiente $\mathrm{M}_{1}$ )

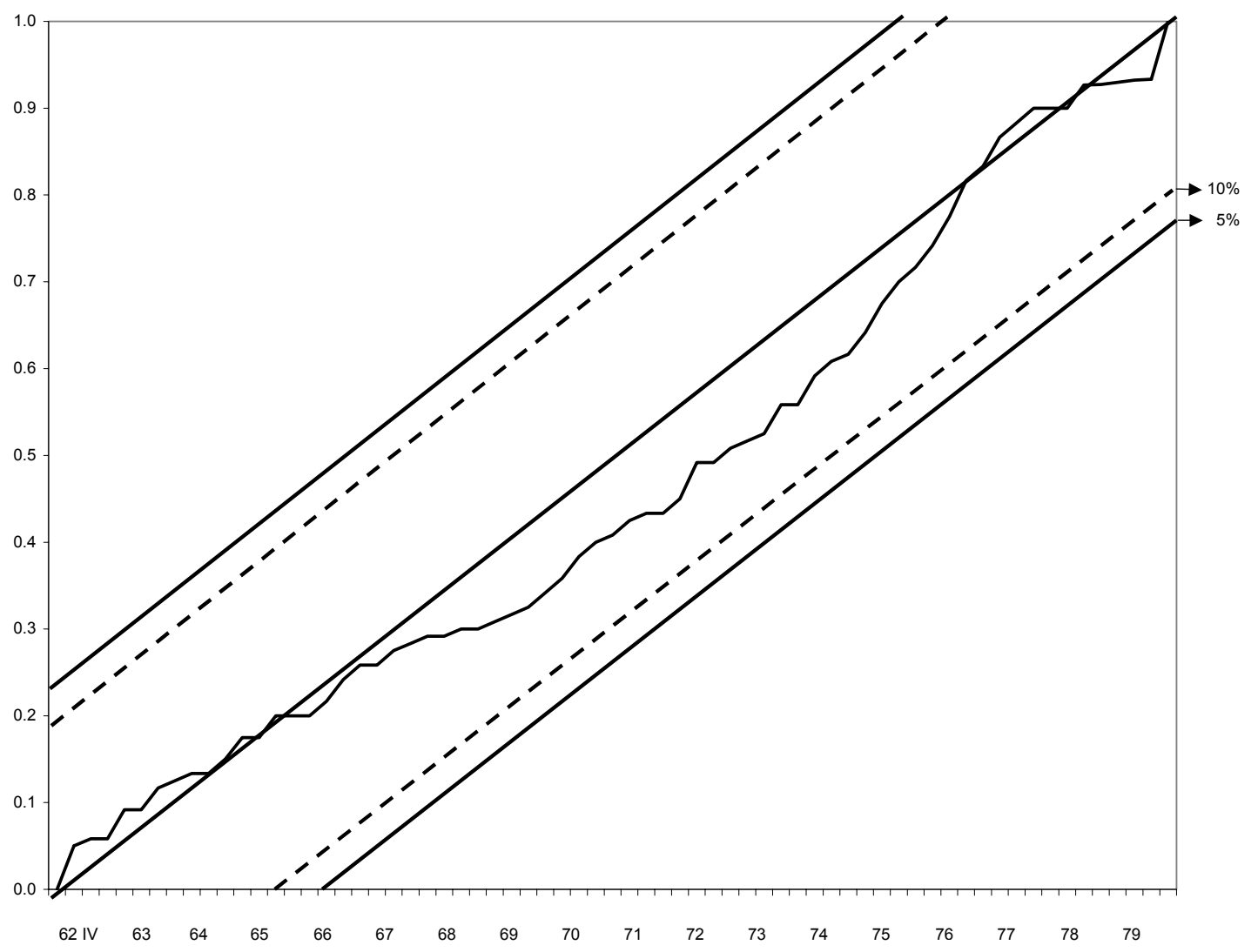

Como puede apreciarse, la hipótesis de estabilidad no puede ser rechazada ni siquiera a un nivel de significación del 10 por ciento. Aún, más, no se observan movimientos bruscos de $\mathrm{S}_{r}$ ni en 1973 -año en que comenzó la inflación-, ni en el período

\footnotetext{
1/ La definición de Co, así como su distribución, están explicados en Durbin (1969). También los valores
} críticos de la prueba para distintos niveles de significación están dados en el cuadro 1 de este artículo. 
posterior a la devaluación de 1976. Según la gráfica, es en los años de 1968-1970 cuando se localizan los mayores movimientos para $\mathrm{S}_{r}$.

Esta prueba se realizó suponiendo que el coeficiente de autocorrelación se mantenía constante para todo el período muestral, con el objeto de corregir la estimación por la presencia de correlación seriada de primer orden. Así, se transformaron las variables de la ecuación (1) sustituyendo el valor de cada una de ellas por el producto del coeficiente de autocorrelación y del valor de la misma variable rezagada un período. Es decir,

$$
\mathrm{X}_{t}=\mathrm{X}_{t}-\hat{\rho} \mathbf{X}_{t-1}
$$

donde $\mathrm{X}_{t}$ representa el valor de la variable transformada, $\mathrm{x}_{t}$ el de la observada $\mathrm{y} \rho$ el coeficiente de correlación estimado ${ }^{1 /}$.

\footnotetext{
${ }^{1 /}$ Se utilizó el coeficiente $\rho$ del cuadro 1 para el período 1960-1972. Asimismo, se realizó un análisis de los residuales de la ecuación 1 con el objeto de verificar la existencia de correlación seriada de orden mayor a la unidad. El análisis, efectuado por el método de Box-Jenkins sobre 24 rezagos, no permite rechazar la hipótesis de que dicha serie es ruido blanco a niveles altos de significación. El valor calculado de la distribución de los rezagos fue de 10.90 , que debe ser comparado con una $\mathrm{X}^{2}$ con 24 grados de libertad. A un nivel de significación de 25 por ciento, $X^{2}(24)=28.24$
} 


\section{Resultados para $\mathrm{M}_{2}$.}

La ecuación (2) corresponde a la mejor estimación de demanda de dinero en sentido amplio $\left(\mathrm{M}_{2}\right)$ efectuada en el trabajo mencionado anteriormente.

$$
\begin{aligned}
\ln \left(\frac{M_{2}}{P}\right) & =\beta_{0}+\beta_{1} \ln \mathrm{Y}_{t}^{c}+\beta_{2} \ln \mathrm{RNL}_{t}+\beta_{3} \ln \pi_{2}+\beta_{4} \mathrm{CME}_{t} \\
& +\beta_{5} \ln \left(\frac{M_{2}}{P}\right)_{t-1}+\mathrm{d}_{1}+\mathrm{d}_{2}+\mathrm{d}_{3}
\end{aligned}
$$

donde $\mathrm{CME}=\frac{\text { Pasivos no Monetarios no Liquidos en Moneda Extranjera }}{\text { Pasivos no Monetarios no liquidos totales }}, \mathrm{y}$ la de función de $\mathrm{M}_{2}$ es:

$\mathrm{M}_{1}+$ Pasivos no Monetarios en Moneda Nacional. El cuadro 6 reproduce los resultados de estimar la ecuación (2).

\section{CUADRO 6}

Estimaciones de la Ecuación 2

Variable dependiente $\mathrm{M}_{2} / \mathrm{P}$

\begin{tabular}{lccccccccc}
\hline PERIODO & $\mathrm{Y}_{t}^{c}$ & $\mathrm{RNL}_{t}$ & $\pi_{t-1}$ & $\mathrm{CME}$ & $\mathrm{m}_{t-1}$ & $\mathrm{R}^{2}$ & $\begin{array}{c}\text { Durbin } \\
\mathrm{h}\end{array}$ & $\begin{array}{c}\text { Error } \\
\text { Rho }\end{array}$ \\
\hline $1960-1972$ & $.081^{*}$ & $-.166^{*}$ & $-.003^{*}$ & $-.206^{*}$ & $.880^{*}$ & .999 & .861 & -.10 & .0120 \\
& $(1.69)$ & $(-4.61)$ & $(-2.08)$ & $(-3.82)$ & $(23.6)$ & & & & \\
$1960-1976$ III & $.102 *$ & $-.113^{*}$ & $-.008^{*}$ & $-.213^{*}$ & $.860^{*}$ & .999 & 1.07 & -.24 & .0152 \\
& $(2.30)$ & $(-3.62)$ & $(-2.03)$ & $(-4.29)$ & $(24.2)$ & & & & \\
$1960-1979$ & $.205^{*}$ & $-.069 *$ & $-.001 *$ & $-.078^{*}$ & $.844 *$ & .997 & 1.24 & -.31 & .0235 \\
& $(4.04)$ & $(-1.84)$ & $(-5.61)$ & $(-1.72)$ & $(21.4)$ & & & & \\
\hline
\end{tabular}

Fuente: G. Ortiz (1980), Cuadro 14.

Todos los coeficientes muestran los signos esperados y son significativos a un nivel de 5\%. Después de varias pruebas, la variable CME se incluyó como "proxy" para 
medir expectativas de devaluación en sustitución de la variable dtc utilizada para $\mathrm{M}_{1}$, debido a que produce mejores resultados para $\mathrm{M}_{2}$.

Comparando los cuadros 1 y 2 , se observa que los coeficientes correspondientes a las regresiones de $\mathrm{M}_{2}$ permanecen bastante estables entre los períodos 1960-1972 y 1960.1976 III, pero se modifican drásticamente cuando se pasa al último período que incluye la devaluación de 1976 y la etapa de contracción financiera que el siguió. Así, mientras que la elasticidad con respecto al rendimiento se redujo drásticamente. Por otra parte, el error estándar de la regresión se incrementó también de manera sustancial en la última estimación con respecto a las dos primeras.

Este comportamiento de las ecuaciones estimadas sugiere la presencia de un cambio estructural de los coeficientes. Por ello, se realizó una prueba de Chow similar a la aplicada en la ecuación 1, encontrándose que se rechaza la hipótesis nula de igualdad de los coeficientes en los períodos 1960-1976 III y 1960-1979.

Por otra parte, se realizaron simulaciones estáticas y dinámicas de la ecuación (2) para el período 1973-I a 1979-IV que se presentan en las gráficas 5 y 6. 
GRAFICA 5

Simulación Estática de la Demanda de Dinero $\left(\mathrm{M}_{2}\right)$

(1973-I/1979-IV)

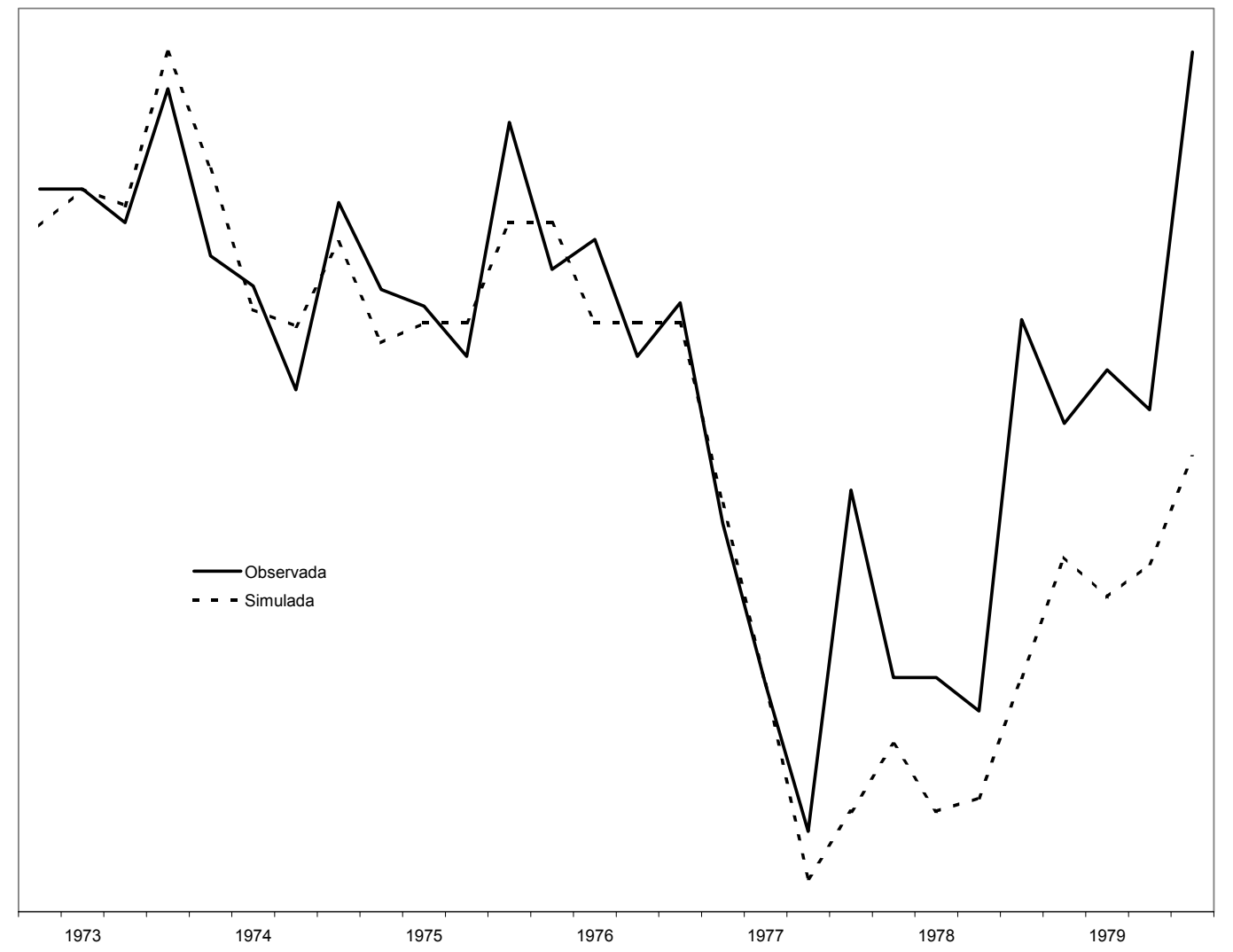

Estadísticas de la Simulación Estática:

Error medio al cuadrado (RMSE)

Error medio absoluto (MAE)

.04071

Error promedio como porcentaje de la variable dependiente

.27790

Coeficiente de desigualdad de Theil

.00277

Fracción del error debido a
i) sesgo
ii) variación
iii) co-variación

.06108

.69240 
Gráfica 6

Simulación Dinámica de la Demanda de Dinero $\left(\mathrm{M}_{2}\right)$

(1973-I/1979-IV)

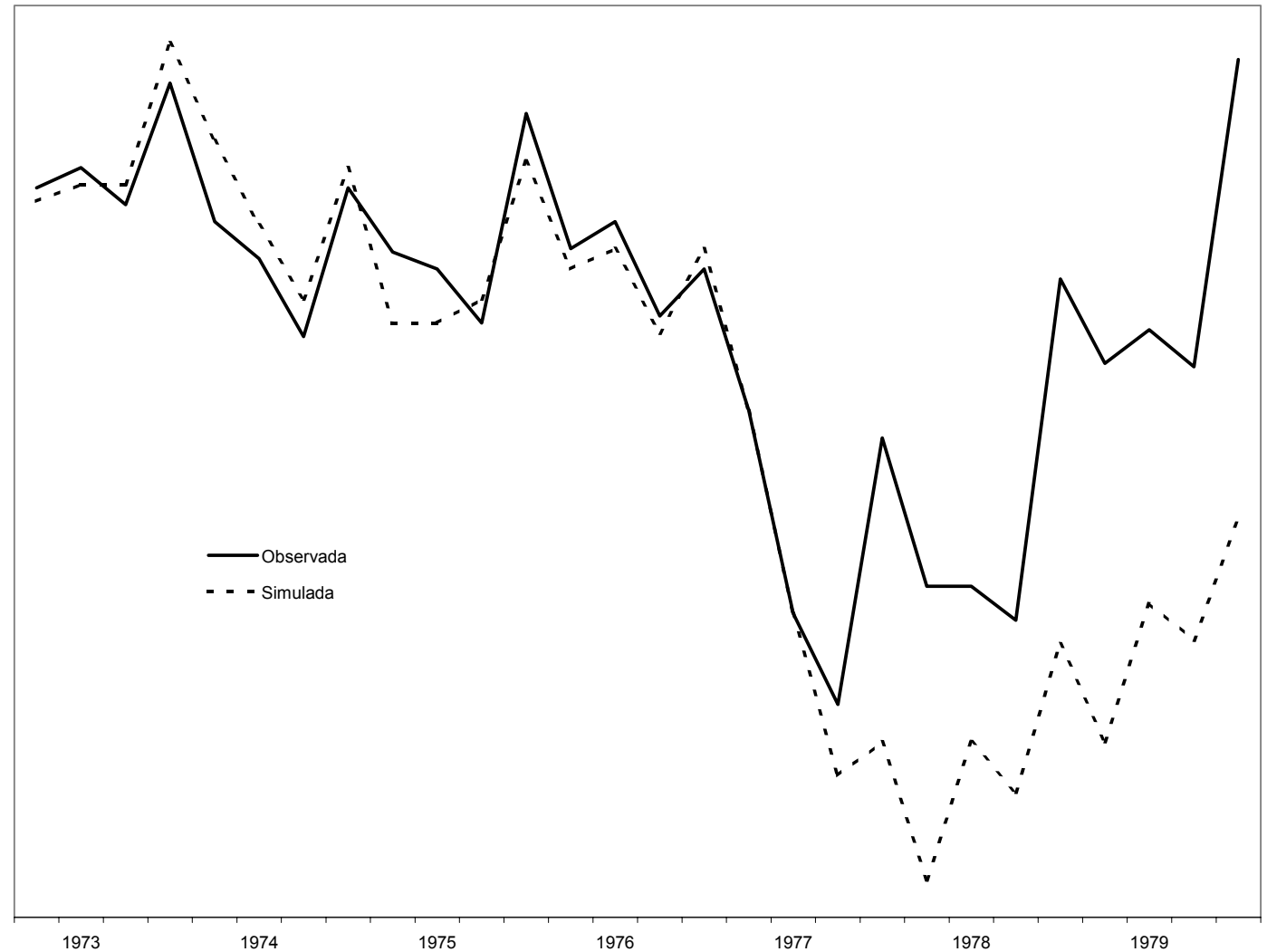

Estadísticas de la Simulación Dinámica:

$\begin{array}{ll}\text { Error medio al cuadrado (RMSE) } & .09317 \\ \text { Error medio absoluto (MAE) } & .06167 \\ \text { Error promedio como porcentaje de la variable dependiente } & .46860 \\ \text { Coeficiente de desigualdad de Theil } & .004172 \\ \text { Fracción del error debido a } & \\ \quad \text { i) sesgo } & .3082 \\ \quad \text { ii) variación } & .1713 \\ \quad \text { iii) co-variación } & .5205\end{array}$

En ambas gráficas se aprecian dos períodos claramente distintos; el primero de 1973 a 1976, y el segundo de 1977 a 1979. Las dos simulaciones, durante el primer período, reproducen fielmente el comportamiento real de $\mathrm{M}_{2}$, al grado de que los errores como porcentaje de la variable dependiente son de .082 y .099 para las proyecciones estáticas y dinámicas, respectivamente. A su vez, en el segundo período, ambas simulaciones subestiman consistentemente los valores observados de la variable 
dependiente, y los errores aumentan considerablemente (a .76 y 1.39 por ciento de $\mathrm{M}_{2}$ para los ejercicios estático y dinámico, respectivamente). El error máximo de la simulación dinámica llega a 2.15 por ciento en el último trimestre de 1979.

Sin embargo, la raíz del error medio al cuadrado (RMSE) es bastante reducida para ambas simulaciones, y no es mucho mayor de la obtenida para $\mathrm{M}_{1}$. Aun mas, los errores promedio como porcentaje de la variable dependiente para todo el período son menores para $\mathrm{M}_{2}$ que para $\mathrm{M}_{1}$.

Por último, en la gráfica 7 se muestra la prueba de Brown, Durbin y Evans realizada para la ecuación 2. Como puede apreciarse, de acuerdo a este procedimiento, no se puede rechazar la hipótesis de estabilidad de los coeficientes a lo largo de todo el período. Aunque este resultado puede sorprender, sobre todo después de que la prueba de Chow realizada indica la presencia de un cambio estructural, debe tenerse en cuenta que el comportamiento de las simulaciones es bastante aceptable aún en el período más inestable ${ }^{\mathrm{1}}$.

\footnotetext{
1/ Un estudio reciente, Cockerline (1979) comparó el poder de la prueba BDE con una prueba de Chow modificada por Riddell (1978), y encontró que para efectos de simulación, esta última prueba es superior en poder y eficiencia a la de Brown, Durbin y Evans.
} 


\section{Gráfica 7}

Prueba de Brown, Durbin y Evans

(Ecuación 2; variable dependiente $\mathrm{M}_{2}$ )

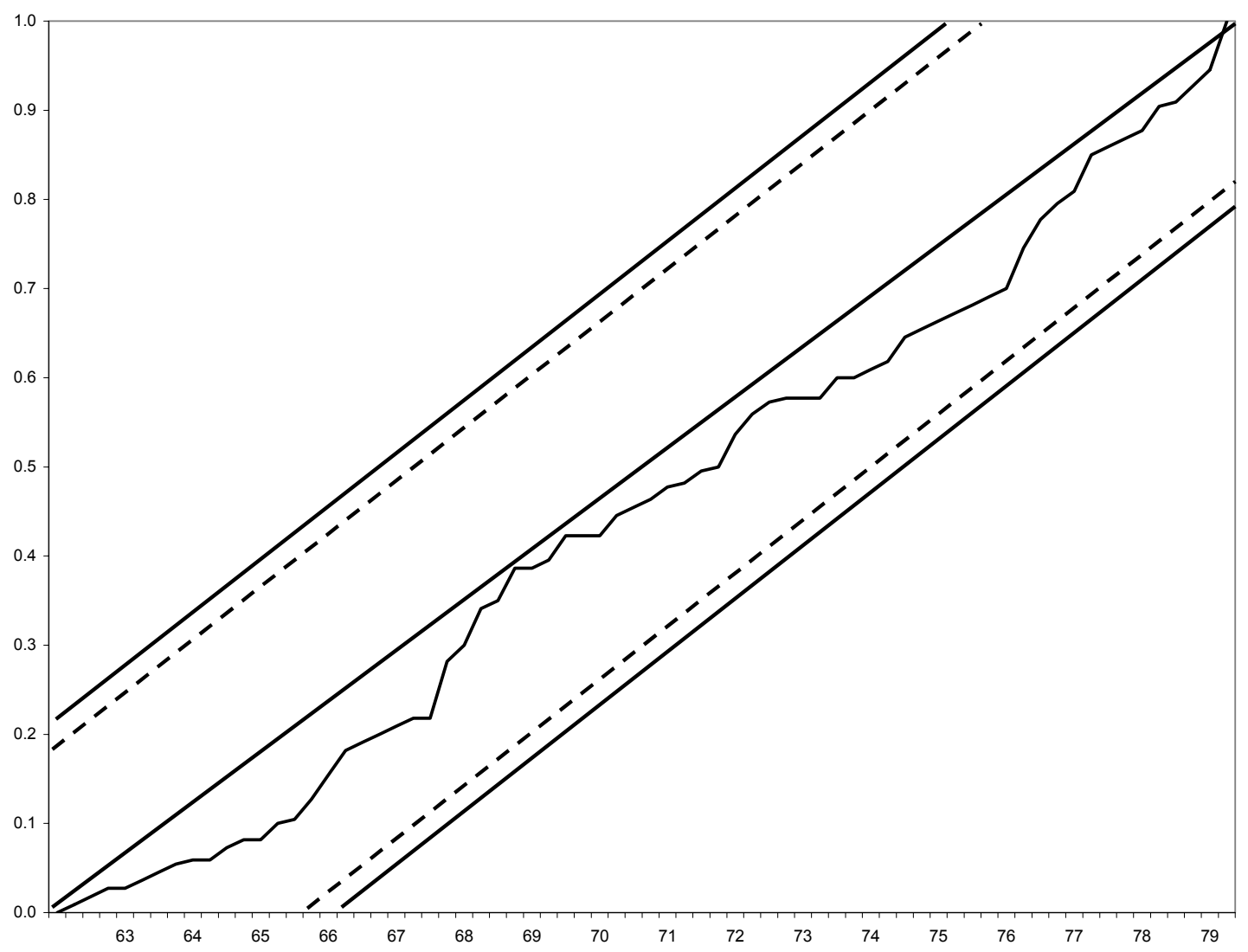

\section{4. $\underline{\text { Conclusiones. }}$}

i) Las diversas pruebas efectuadas indican que la demanda de $M_{1}$ en el período 1960-1979 es una función estable del ingreso, de la tasa de interés pagada sobre pasivos líquidos, de la inflación esperada y del diferencial del tipo de cambio real entre México y Estados Unidos, variable esta última que intenta captar el riesgo cambiario.

ii) Pese a que las propiedades estadísticas de las regresiones que utilizan $\mathrm{M}_{2}$ son, en general, superiores a las correspondientes a $\mathrm{M}_{1}$, las simulaciones estáticas y dinámicas efectuadas con la definición reducida de dinero reproducen con mayor fidelidad el comportamiento de la variable dependiente a partir de 1976. 
iii) La mayor inestabilidad detectada en las simulaciones hechas para $M_{2}$, sugiere que este agregado está mas influenciado por consideraciones de riesgo cambiario que $\mathrm{M}_{1}$. Parece ser que las posibilidades de realizar buenos pronósticos con la ecuación estimada para este último agregado, son bastante buenas. 


\section{B I B L I O G R A F I A}

Brown, R.L., Durbin, J. Y Evans, J.M. (1975), “Techniques for Testing the Constancy of Regression Relationships over Time, with Comments". JRSS, B, No. 2.

Cockerline, J. (1979), "Stability Test and the Demand for Money in Canada, 1956-1977". Proceedings of the Business and Economic Statistic Section. American Statistical Association.

Durbin, J. (1969), "Tests for Serial Correlation in Regression Analysis based on the Periodgram of Least-Squares Residuals". Biometrica, 56, 1.

García, G. Y Pak, S. (1979), "Some Clues in the Case of the Missing Money". American Economic Review. Vol. 69, No. 2, Mayo.

Goldfeld, S. (1973), “The Demand for Money Revisited”. Brookings Papers on Economic Activity: 3.

(1976), "The Case of the Missing Money". Brookings Papers on Economic Activity: 3 .

Hafer, W. Y Hein, E. (1979), "Evidence on the temporal Stability of the Demand for Money Relationship in the United States". Federal Reserve Bank of St. Louis Review, Diciembre.

Hein, E. (1980), "Dynamic Forecasting and the Demand for Money". Federal Reserve Bank of St. Louis Review, Junio/Julio, Vol. 62, No. 6.

Heller, R. Y Khan, M. (1979), “The Demand for Money and the Term Structure of Interest Rates”. JPE, Vol. 82, No. 6, Diciembre.

Johnston, J. (1972), Econometric Models, Techniques and Applications. Prentice Hall, N.J.

Khan, M. (1974), "The Stability of the Demand-for-Money Function in the United States 1901-1965". JPE, Vol. 82, No. 6, Diciembre

Ortiz, G. (1980), "La Demanda de Dinero en México: Primeras Estimaciones". Documento de Investigación No. 28, Subdirección de Investigación Económica, Banco de México, S.A., Septiembre.

Porter, R., Simpson, T. Y Mauskopf, E. (1979), "Financial Innovation and the Monetary Aggregates". Brookings Papers on Economic Activity: 1.

Riddell, W.C. (1978), "The Use of the Stepwise Chow Test”. Research Paper No. 78-2, University of Alberta, Edmonton, Enero. 
BANCO DE MEXICO, S.A.

SUBDIRECCION DE INVESTIGACION ECONOMICA

Serie de Documentos de Investigación

1. ESTRUCTURA FINANCIERA Y EXPERIENCIA CAMBIARIA: MEXICO 19541977.

Guillermo Ortiz. Octubre, 1978.

2. EL FINANCIAMIENTO DEL GASTO PUBLICO EN UNA ECONOMIA EN CRECIMIENTO: EL CASO DE MEXICO.

Alain Ize. Noviembre, 1978.

3. ALGUNOS ASPECTOS DEL ENDEUDAMIENTO PUBLICO EXTERNO EN MEXICO.

Ernesto Zedillo. Diciembre, 1978.

4. UNA APLICACIÓN DEL MODELO BAYESIANO DE DECISION EN EL ANALISIS DE FUNCIONES DE PRODUCCION AGRICOLA.

Héctor E. González Méndez. Diciembre, 1978.

5. POLITICA MACROECONOMICA EN EL CORTO PLAZO: UNA RESEÑA.

Alain Ize. Marzo, 1979.

6. ESTUDIOS DE MONEDA Y BANCA Y POLITICA MONETARIA SOBRE MEXICO: SELECCIÓN BIBLIOGRAFICA DE 1943 A 1978.

Abril, 1979.

7. COMERCIO EXTERIOR MEXICO-ESTADOS UNIDOS: PROBLEMAS DE COMPARABILIDAD ESTADISTICA.

Jorge Carriles Rubio. Mayo, 1979.

8. EXPLOTACION OPTIMA DE RESERVAS PETROLERAS EN UN CONTEXTO MACROECONOMICO.

José Córdoba. Mayo, 1979.

9. ASPECTOS DEFLACIONARIOS DE LA DEVALUACION DEL PESO MEXICANO DE 1976.

José Córdoba y Guillermo Ortiz Mayo, 1979.

10. EXTRACCION OPTIMA DE PETROLEO Y ENDEUDAMIENTO EXTERNO: EL CASO DE MEXICO.

Ernesto Zedillo. Junio, 1979.

11. IMPUESTOS DIRECTOS: PROGRESIVIDAD OPTIMA. Jesús Seade. Septiembre, 1979. 
12. OPCIONES DE POLITICA ECONOMICA 1979-1982.

Sócrates Rizo y Leopoldo Solís. Septiembre, 1978.

13. INTERMEDIARIOS FINANCIEROS Y MERCADOS IMPERFECTOS DE CAPITAL.

Guillermo Ortiz. Septiembre, 1979.

14. ESTIMACIONES DE EQUILIBRIO GENERAL DE LOS EFECTOS DE LAS DISTORSIONES EN LOS MERCADOS DE FACTORES: EL CASO DE MEXICO. José Sidaoui y Richard H. Sines. Octubre, 1979.

15. UN ANALISIS DE LA INFLACION EN MEXICO.

Alain Ize. Octubre, 1979.

16. ANALISIS DE LOS COMPONENTES DEL CAMBIO ESTRUCTURAL CON UN MODELO DE EQUILIBRIO GENERAL, 1970-75.

José Sidaoui y Richard H. Sines. Enero, 1980.

17. TIPOS DE CAMBIO FLOTANTES Y DESLIZ CAMBIARIO: LAS EXPERIENCIAS DE ALGUNOS PAISES EN DESARROLLO.

Guillermo Ortiz y Leopoldo Solís. Enero, 1980.

18. UN MODELO DE INFLACION Y CRECIMIENTO EN UNA ECONOMIA CAPITALISTA EN DESARROLLO.

Alain Ize. Enero, 1980.

19. CRECIMIENTO E INFLACION: ALTERNATIVAS CAMBIARIAS PARA MEXICO.

Guillermo Ortiz y Leopoldo Solís. Febrero, 1980.

20. COMPORTAMIENTO DE LA CAPTACION BANCARIA EN MEXICO.

Héctor E. González Méndez. Mayo, 1980.

21. LA ENCUESTA DE TURISMO RECEPTIVO, REPORTE METODOLOGICO.

Alberto Vargas Aguayo. Junio, 1980.

22. AJUSTE ESTACIONAL DE UNA SERIE DE TIEMPO MEDIANTE EL USO COMPLEMENTARIO DE METODOS TRADICIONALES Y LA TECNICA DE BOX-JENKINS.

Gabriel Vera Ferrer y Víctor M. Guerrero. Junio, 1980.

23. DISTRIBUCION DEL FINANCIAMIENTO OTORGADO POR EL SISTEMA BANCARIO MEXICANO A LA BANCA PRIVADA Y MIXTA.

Víctor M. Guerrero y Gabriel Vera Ferrer. Julio, 1980. 
24. LA MIGRACION INDOCUMENTADA A ESTADOS UNIDOS: UN NUEVO ENFOQUE.

Juan Díez-Canedo. Julio, 1980.

25. UN MODELO FINANCIERO DE DESEQUILIBRIO A CORTO PLAZO PARA LA ECONOMIA MEXICANA.

Alain Ize. Julio, 1980.

26. ESTIMACION DE LA FUNCION DE IMPORTACIONES PARA MEXICO.

Javier Salas. Agosto, 1980.

27. UNA ALTERNATIVA PARA LA MEDIA ARITMETICA EN EL CALCULO DE PROMEDIOS SIMPLES DE RELATIVOS DE PRECIOS: LA MEDIA GEOMETRICA.

Gabriel Vera Ferrer y Víctor M. Guerrero. Agosto, 1980.

28. LA DEMANDA DE DINERO EN MEXICO: PRIMERAS ESTIMACIONES.

Guillermo Ortiz. Septiembre, 1980.

29. ECONOMIAS DE ESCALA Y CONCENTRACION BANCARIA: EL CASO DE MEXICO.

Héctor E. González Méndez. Octubre, 1980.

30 LA ESTABILIDAD DE LA DEMANDA DE DINERO EN MEXICO.

Guillermo Ortiz. Noviembre, 1980. 